ARTICLE HISTORY: Received: August: 7, 2021 Accepted: September 28, 2021 Published: October 4, 2021

ИМИТАЦИОННОЕ МОДЕЛИРОВАНИЕ ЦИФРОВЫХ АНТЕННЫХ РЕШЕТОК

Нечаев Юрий Борисович

доктор физико-математических наук, профессор

Пешков Илья Владимирович

кандидат физико-математических наук, доцент

Фортунова Наталия Александровна

кандидат технических наук доцент

Зайцева Ирина Николаевна

кандидат педагогических наук, доиент

Елецкий государственный университет им. И.А. Бунина

\title{
DIGITAL ANTENNA ARRAYS SIMULATION
}

\author{
Nechaev Yuriy Borisovich \\ Doctor of Physical and Mathematical Sciences, Professor \\ Peshkov Ilya Vladimirovich \\ Candidate of Physical and Mathematical Sciences, Associate Professor \\ Fortunova Natalia Alexandrovna \\ Candidate of Technical Sciences Associate Professor \\ Zaitseva Irina Nikolaevna \\ candidate of pedagogical sciences, associate professor \\ Yelets State University named after I.A. Bunin
}

\begin{abstract}
Аннотация. В статье приведено описание имитационной модели цифровой антенной решетки, которая может использоваться в системах передачи широкополосных сигналов под воздействием широкополосных помех. Исходный информационный сигнал имеет скорость 19200 бит/с для потока I и Q. Доказано, что уровень сигнала после применения диаграммообразования цифровой антенной решетки в четыре раза превосходит уровень сигнала без его использования, что подтверждает преимущество цифровых радиотехнических систем.

Abstract. The article describes a simulation model of a digital antenna array, which can be used in broadband signal transmission systems under the influence of broadband interference. The initial information signal has a speed of $19200 \mathrm{bit} / \mathrm{s}$ for the I and Q stream. It is proved that the signal level after applying the diagramming of a digital antenna array is four times higher than the signal level without its use, which confirms the advantage of digital radio systems.

Ключевые слова: цифровая антенная решетка, диаграмма направленности, диаграмма образования, имитационное моделирование.

Key words: digital antenna array, directional diagram, education diagram, simulation.

Перспективным типом антенных устройств следует признать ЦАР, подтверждением чему служат многочисленные публикации [1-3]. Для реализации высоких возможностей таких систем приемные каналы многолучевой диаграммо-образующей схемы (ДОС) должны обладать идентичными амплитудно-фазовыми и частотными характеристиками, поскольку "любая система, в которой хотя бы частично процесс формирования луча осуществляется вне первичных приемных каналов, чувствительна к фазовым и амплитудным ошибкам в приемных каналах и в антенне" [4]. В отношении фазированных антенных решеток (ФАР) наблюдается аналогичная проблема. Но цифровой метод формирования лучей в ЦАР способствует более эффективной компенсации погрешностей в амплитудно-фазовом распределении поля на раскрыве антенны без жестких ограничений "в абсолютных допусках на параметры приемных каналов и использования контроля по цепям обратной связи" [5].

На рис. 1 показана упрощенная блок-схема устройства цифрового формирования диаграммы направленности (ДН). Приведённая приёмная $N$-элементная антенная решетка, сигналы с элементов которой после прохождения приемника в соответствующем канале умножаются на коэффициенты весового вектора $\overrightarrow{\boldsymbol{w}}^{H}$, где $(\cdot)^{\mathrm{H}}$ - комплексно-сопряженное транспонирование, а затем суммируются.
\end{abstract}




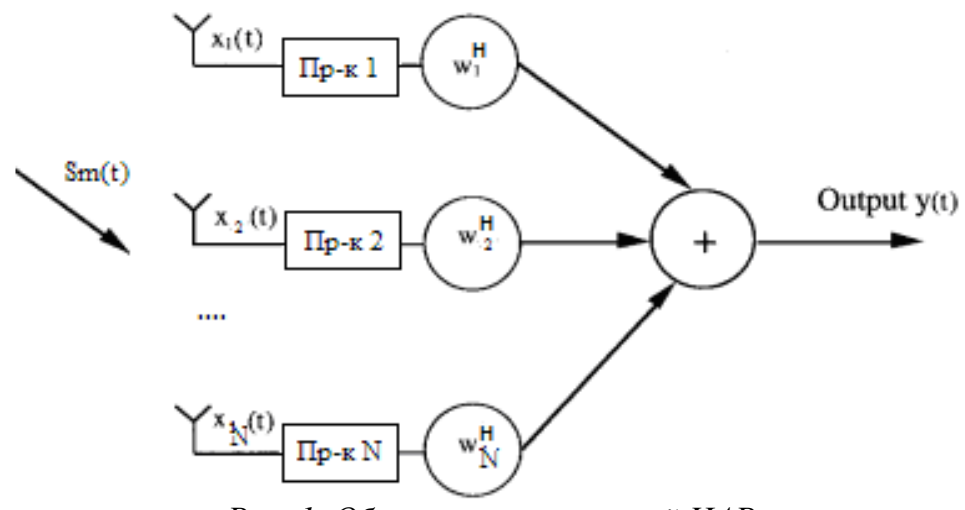

Рис. 1. Общая схема приемной ЦАР

Далее приведено описание физической структуры цифровой антенной решетки (рис. 2) для работы в СВЧдиапазоне [6].

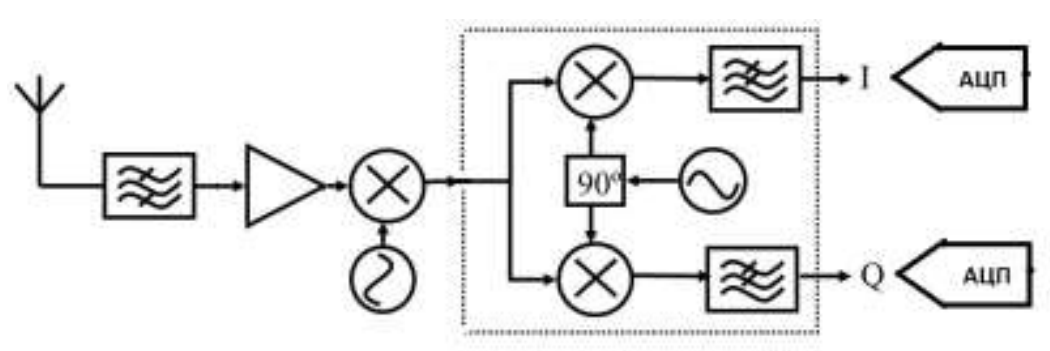

a)

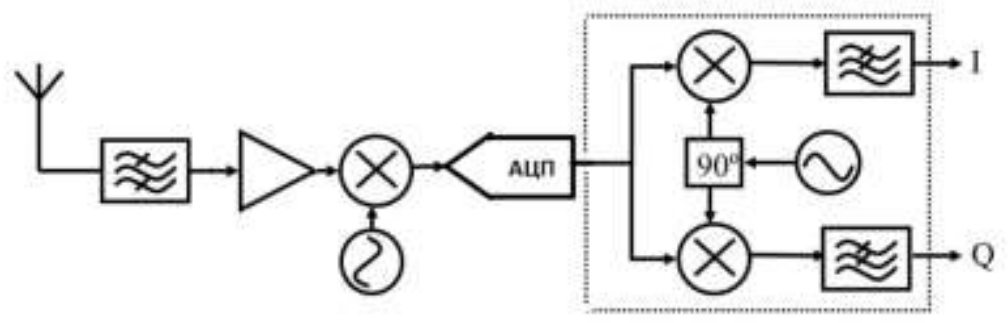

б)

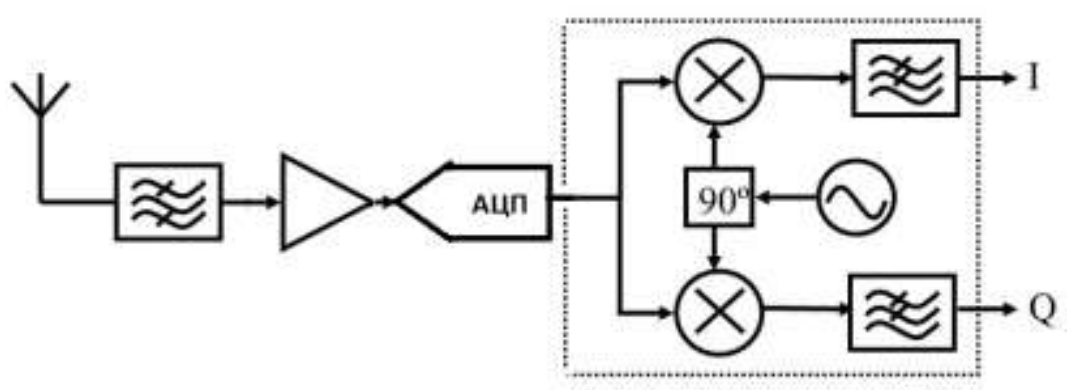

в)

Рис. 2. Типы размещения АЦП в приемниках ЦАР

В структуре ЦАР по месту размещения аналого-цифрового преобразователя можно определить три типа приемников: оцифровка сигнала на низкой частоте (рис. 2,a), оцифровка сигнала на промежуточной частоте (рис. 2,б) и оцифровка сигнала на радиочастоте (рис. 2,в).

Демодулированные данные в цифровой форме поступают в модуль сбора и временного хранения принятых данных. Для обработки информации от нескольких каналов и реализации управления системой в целом в масштабе реального времени применение ПЛИС выглядит наиболее оптимальным вариантом. 
Была разработана модель цифровой антенной решетки, которая может использоваться в системах радиосвязи при приеме широкополосных сигналов под воздействием широкополосных помех. Технический результат заключается в увеличении отношения сигнал / (помеха + шум) при обработке широкополосных сигналов относительно помеховых сигналов независимо от их ширины полосы частот и мощности в любой среде помехового сигнала. Адаптивная антенная решетка содержит шесть антенных элементов (рис.2), на выходах которых имеется шесть аналоговых приемников (на рис. 3 - «1»), состоящих из малошумящих усилителей, микшеров, полосовых фильтров и аналоговых генераторов синфазные и квадратурные компоненты. Затем сигналы попадают в блок аналого-цифрового преобразования (на рис.3 - «2»), а также в цифровой понижающий преобразователь на основе фильтра приподнятого косинуса (на рис.3 - «3»).

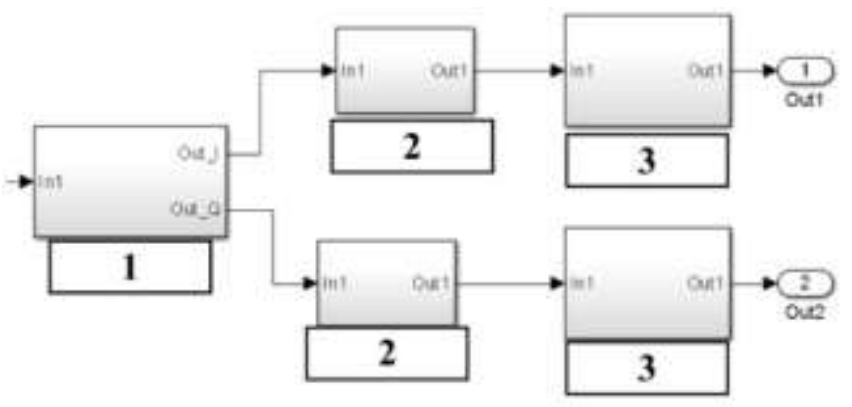

Рис. 3. Блок-схема одного канала

На рис.4 показана схема системы приемной цифровой антенной решетки. Оцифрованный сигнал поступает в блок формирования обратной корреляционной матрицы (на рис. 4 - «1») и вычисления пространственных координат источников сигнала (на рис. 4 - «2»). Далее рассчитанные значения угловых координат передаются в блок для формирования цифровой диаграммы направленности (на рис. 3 - «3»). Этот блок компенсирует запаздывание фазы в каждом канале антенной решетки, а затем когерентно складывает все шесть сигналов для получения более мощного выходного сигнала. В практических приложениях истинная матрица обычно неизвестна и вместо этого используется оценка ковариационной матрицы, полученной из набора из $K$ временных выборок. Обратная корреляционная матрица вычисляется и обновляется в соответствии со следующей формулой, для определения сложного вектора сигнала, генерируемого из каждого выхода:

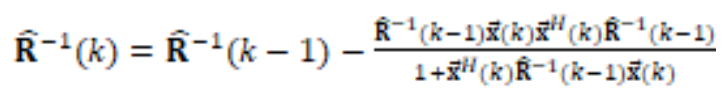

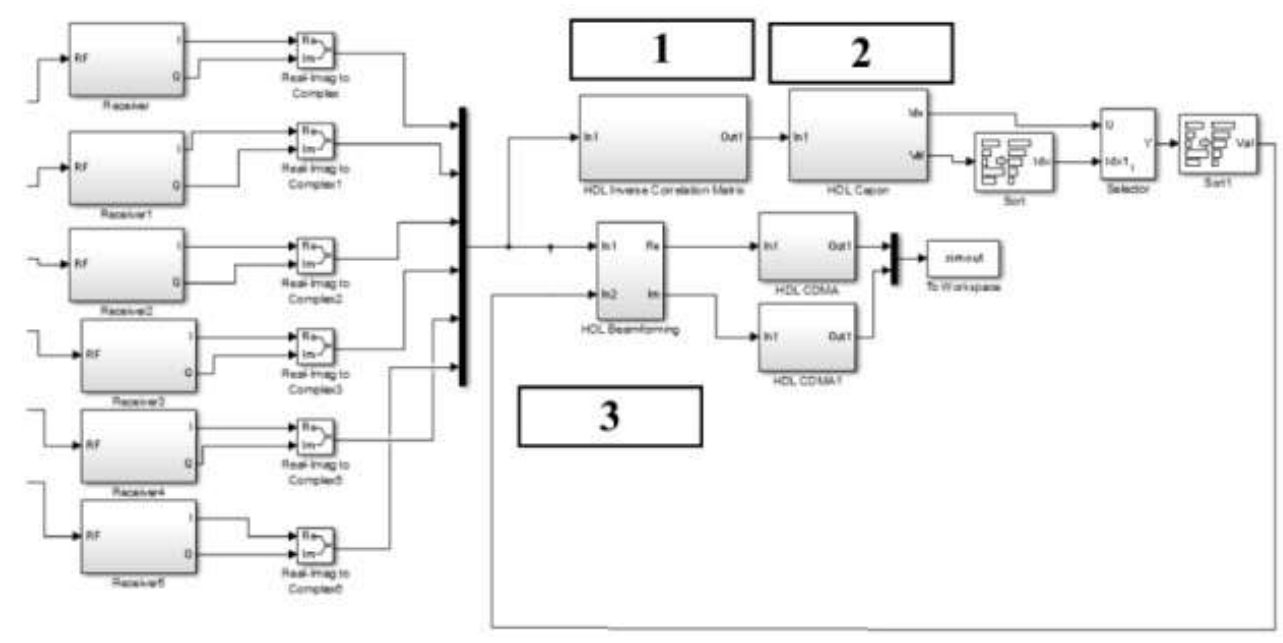

Рис. 4. Структурная схема ЦАР

Таким образом, полученная матрица переходит в следующий блок 2, где вычисление координат источников сигналов выполняется по следующей формуле: 


$$
P(\theta)=\frac{1}{\vec{a}^{\vec{n}}\left[(\theta) \mathbf{R}^{-1} \overrightarrow{\mathbf{z}} \mid \theta\right)}
$$

Если текущий угол $\theta$ вектора совпадает с направлением на любой источник сигнала $\left\{\theta_{\mathrm{m}}\right\}_{\mathrm{m}=1}^{M}$, тогда в этой точке функция $P(\theta)$ имеет особенность типа $0^{-1}$. Пики функции определяют угловые положения источников сигнала.

В телекоммуникационных системах выходной сигнал в момент времени $k$ получается путем линейной комбинации данных с $N$ выходов антенных элементов:

$$
y(k)=\overrightarrow{\mathbf{w}}^{H} \overrightarrow{\mathbf{x}}(k)
$$

где $\overrightarrow{\boldsymbol{w}}$ - вектор весовых коэффициентов. Если изменять коэффициенты $\overrightarrow{\boldsymbol{w}}$ можно ориентировать основной лепесток диаграммы направленности в любом направлении и адаптивно управлять ее формой, чтобы общая мощность помех и аддитивный шум были минимальными с минимальными искажениями полезного сигнала, T.e.

$$
\min _{\vec{W}} E\left\{\overrightarrow{\mathbf{w}}^{H} \overrightarrow{\mathbf{x}}_{\mathrm{i}+\mathrm{n}}\right\} \text { и } \overrightarrow{\mathbf{w}}^{H} \overrightarrow{\mathbf{a}}_{1}=1
$$

где $\overrightarrow{\boldsymbol{x}}_{i+n}$ сигнал от с выходов элементов антенной решетки, содержащий только помехи и шум. В этом формирователе луча вектор весовых коэффициентов выбирается равным вектору направления полезного сигнала, т.е.:

$$
\overrightarrow{\mathbf{w}}=\overrightarrow{\mathbf{a}}\left(\theta_{1}\right)
$$

Чтобы проиллюстрировать работу полученной модели цифровой антенной решетки, мы сформируем сигнал QPSK на ее входе. Исходный информационный сигнал имеет скорость 19200 бит / с для потоков I и Q. Затем каждый бит модулируется псевдослучайной последовательностью, длительность которой составляет 63 бита. Таким образом, получается, что каждый канал имеет конечную скорость 1209600 бит / с. Направление к источнику оценивается, и формирование диаграммы направленности выполняется в цифровой части разработанной приемной антенной решетки. После этого проводится пространственная фильтрация [7]. На рис. 5, 6 представлены диаграммы уровня сигналов.

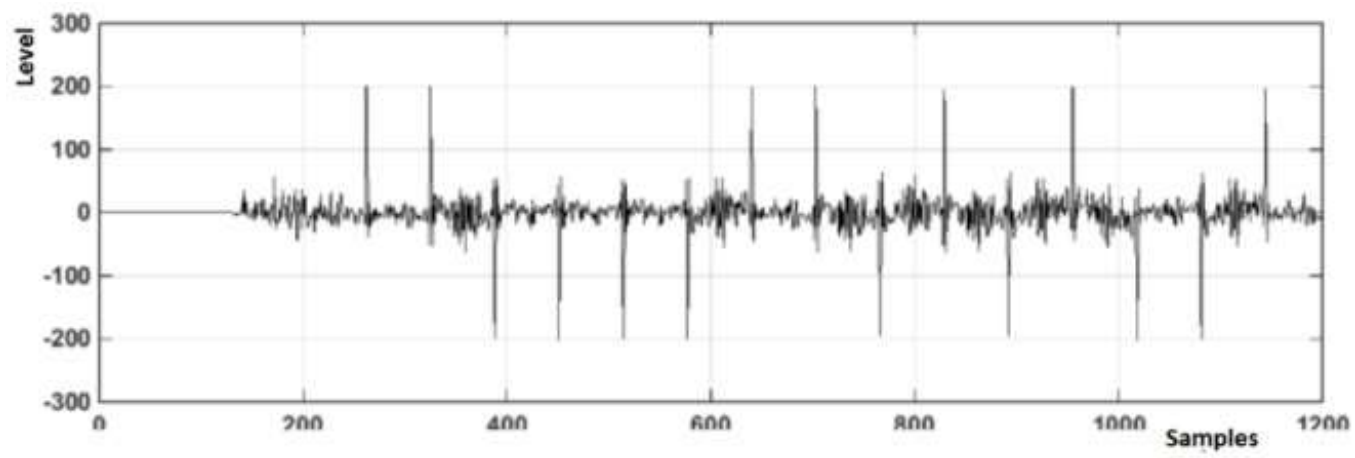

Рис. 5. Сигнал после цุифрового формирования луча и согласованной фильтрации 


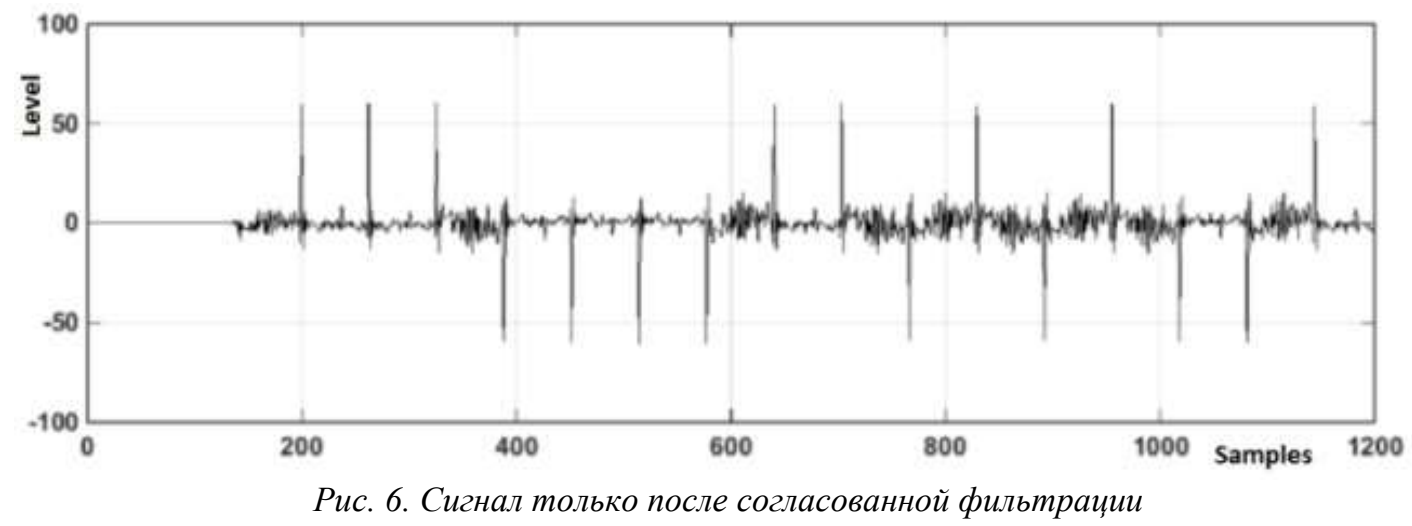

Как видно из рисунков 5,6 уровень сигнала после применения формирования диаграммы направленности цифровой антенной решетки в четыре раза выше, чем без ее использования, что подтверждает преимущество цифровых систем радиосвязи.

\section{Список использованных источников}

1. Nechaev, Yuri B.; Peshkov, Ilia W.. Measuring of False Peaks Occurring via Planar Antenna Arrays DOA Estimation. International Journal of Advances in Telecommunications, Electrotechnics, Signals and Systems, [S.1.], v. 6, n. 2, p. 45-51, may 2017.

2. Нечаев Ю.Б. Оценка границы Крамера-Рао для 2D радиопеленгации в плоских антенных решетках / Ю.Б. Нечаев, И.В. Пешков// Вестник НТУ «КПИ». Серия Радиотехника, Радиоаппаратостроение. - 2016. Выпуск 67. - с. 12-17

3. Нечаев Ю.Б., Пешков И.В. Пространственная обработка сигналов цифровыми антенными решетками: монография. - Елец: ЕГУ, 2016. - 90 с.

4. Воскресенский Д. И., Гостюхин В. Л., Максимов В. М., Пономарев Л. И. Устройства СВЧ и антенны/Под ред. Д. И. Воскресенского. Изд. 2-е, перераб. и доп. Москва: Радиотехника, 2006. 376 с. 8

5. Ahn H., Tomasic B., Liu Sh. Digital beamforming in a large conformal phased array antenna for satellite operations support -Architecture, Design and Development//IEEE Intern. Symposium on Phased Array Systems and Technology, Boston, Massachusetts, USA, October 2010. -2010. -P. 423-431.

6. Нечаев Ю.Б. Автоматическое калибрование для имитационной модели и экспериментального образца адаптивной антенной решетки / Ю.Б. Нечаев, И.В. Пешков // Теория и техника радиосвязи. - 2015. - № 3. - с. 70 -82 .

7. Nechaev Yu.B. The multibeam digital antenna array with directive emitters for pseudo noise (PN) signals spatial filtering / Yu.B. Nechaev ; I.W. Peshkov ; N.A. Fortunova // 2019 Systems of Signal Synchronization, Generating and Processing in Telecommunications (SYNCHROINFO). (IEEE \# 47541). - Moscow Technical University of Communication and Informatics (MTUCI). Yaroslavl, Russia

\section{References}

1. Nechaev, Yuri B.; Peshkov, Ilia W.. Measuring of False Peaks Occurring via Planar Antenna Arrays DOA Estimation. International Journal of Advances in Telecommunications, Electrotechnics, Signals and Systems, [S.1.], v. 6, n. 2, p. 45-51, may 2017.

2. Nechaev Y.B. Estimation of the Cramer-Rao boundary for 2D radio direction finding in flat antenna arrays / Yu.B. Nechaev, I.V. Peshkov // Vestnik NTU «KPI». Seriya Radiotekhnika, Radioapparatostroyeniye. - 2016. - Issue 67. - p. $12-17$

3. Nechayev YU.B., Peshkov I.V. Prostranstvennaya obrabotka signalov tsifrovymi antennymi reshetkami: monografiya. - Yelets: YEGU, 2016. - 90 p.

4. Voskresenskiy D. I., Gostyukhin V. L., Maksimov V. M., Ponomarev L. I. Ustroystva SVCH i antenny/Pod red. D. I. Voskresenskogo. Ed. 2nd, rev. and add. Moscow: Radiotekhnika, 2006. 376 p. 8

5. Ahn H., Tomasic B., Liu Sh. Digital beamforming in a large conformal phased array antenna for satellite operations support -Architecture, Design and Development//IEEE Intern. Symposium on Phased Array Systems and Technology, Boston, Massachusetts, USA, October 2010. -2010. -P. 423-431.

6. Nechaev Y.B. Automatic calibration for a simulation model and an experimental prototype of an adaptive antenna array / Yu.B. Nechaev, I.V. Peshkov // Teoriya i tekhnika radiosvyazi. - 2015. - № 3. - pp. 70 - 82.

7. Nechaev Yu.B. The multibeam digital antenna array with directive emitters for pseudo noise (PN) signals spatial filtering / Yu.B. Nechaev ; I.W. Peshkov ; N.A. Fortunova // 2019 Systems of Signal Synchronization, Generating and 
Processing in Telecommunications (SYNCHROINFO). (IEEE \# 47541). - Moscow Technical University of Communication and Informatics (MTUCI). Yaroslavl, Russia 